\title{
Noise-reversed stability of Turing patterns versus Hopf oscillations near codimension-two conditions
}

\author{
Sergio Alonso ${ }^{1}$ and Francesc Sagués ${ }^{2}$ \\ ${ }^{1}$ Physikalisch-Technische Bundesanstalt, Abberstrasse 2-12, 10587 Berlin, Germany \\ ${ }^{2}$ Departament de Química Física, Universitat de Barcelona, 08028 Barcelona, Spain
}

(Received 24 July 2009; published 21 September 2009)

\begin{abstract}
Pattern formation induced by noise is a celebrated phenomenon in diverse reaction-diffusion systems. Here we report numerical simulations with the Lengyel-Epstein model for the chlorine dioxide-iodine-malonic acid reaction when perturbed with an external spatiotemporal stochastic forcing in the vicinity of the Hopf and Turing codimension-two bifurcation. Competition between Turing and Hopf modes gives rise to the generation of transient Turing patterns evolving to stationary global oscillations. This situation is reversed by the introduction of external fluctuations and Turing patterns become dominant in this case. The increase in the spatial coherence is found for intermediate noise intensity and small correlation length.
\end{abstract}

DOI: 10.1103/PhysRevE.80.035203

PACS number(s): 82.40.Bj, 05.40.Ca, 82.40.Ck, 47.54.-r

Spatially extended systems may display different modes of self-organization. Most common under reaction-diffusion conditions are Hopf-oscillatory (spatially uniform) regimes and Turing-patterned (time-steady) structures [1]. Situations where both might coexist had been envisaged for a long time although clear experimental evidences came only very recently $[2,3]$. Above, but not far from codimension-two conditions, one could interestingly look for a scenario of HopfTuring competition. In this respect, a striking mechanism of mode selection is here reported based on the use of spatiotemporal noise with controlled strength and correlation parameters. In particular we demonstrate the dominance of Turing patterns over Hopf regimes under moderate random forcing of small correlation length.

As a matter of fact, the possibility to order a system from noise should not come as a surprise any more. A variety of experimental and numeric realizations of such counterintuitive paradigm have accumulated during these last years, as recently reviewed [4]. The Hopf to Turing noise-reversed stability adds to the by now best known scenarios, from noise-induced phase transitions to noise-mediated resonances, to better assess the role of fluctuations in exploiting nonlinearities to ultimately structure an spatially extended system.

Our Rapid Communication refers to a chemical reactiondiffusion system, one of the most appropriate contexts in which to look for pattern tuning under external deterministic stimuli $[5,6]$. This possibility of control extends to random forcing as proved for wave patterns in the BelousovZhabotinsky system $[7,8]$ and for some reactions in catalytic surfaces [9]. Studies of noise-induced Turing patterns, far from other instabilities, have been also reported [10-12]. Here, differently, we concentrate on the effects of noise on competing modes $[13,14]$. We pursue our previous analysis [13] reporting, both in experiments and numeric simulations, a situation of noise-arrested oscillations in mixed TuringHopf patterns of the photosensitive chlorine dioxide-iodinemalonic acid (CDIMA) reaction [15]. This time our purely numeric analysis encompasses a wider area, farther from the codimension-two point, in the phase diagram of the corresponding Lengyel-Epstein (LE) model [16]

$$
\begin{gathered}
\frac{\partial u}{\partial t}=a-c u-\frac{4 u v}{1+u^{2}}-\phi(\vec{x}, t)+\nabla^{2} u, \\
\frac{\partial v}{\partial t}=b\left(c u-\frac{u v}{1+u^{2}}+\phi(\vec{x}, t)+d \nabla^{2} v\right) .
\end{gathered}
$$

In this dimensionless form, $u$ and $v$ are proportional to the activator $\left(I^{-}\right)$and inhibitor $\left(\mathrm{ClO}_{2}^{-}\right)$concentrations; $a, b, c$ are parameters related to other initial concentrations and rate constants and $d$ denotes the ratio of diffusivities. $\phi(\vec{x}, t)$ stands for the illumination intensity. To mimic experiments with fluctuating illumination, we prescribe $\phi(\vec{x}, t)$ $=\phi_{0}+\xi(\vec{x}, t)$, with $\xi(\vec{x}, t)$ as an additive Gaussian zero-mean random quantity described by the Ornstein-Uhlenbeck statistics

$$
\left\langle\xi(\vec{x}, t) \xi\left(\vec{x}^{\prime}, t^{\prime}\right)\right\rangle=\frac{\epsilon}{\tau} e^{-\left|t-t^{\prime}\right| / \tau} \delta\left(\vec{x}-\vec{x}^{\prime}\right),
$$

where $\epsilon$ stands for the noise strength, whereas $\tau$ introduces the time correlation parameter. The noise is white in space and colored in time and in particular $\tau \rightarrow 0$ recovers the white-noise limit in time and space. The stochastic reactiondiffusion equations (1) were solved using a standard fivepoint formula for the two-dimensional (2D) Laplacian and the Heun method for temporal integration [17]. We consider no-flux boundary conditions.

Figure 1(a) shows the linear stability-based phase diagram of the LE model around the reference state $\left(u_{0}=(a-5 \phi) / 5 c\right.$ and $\left.v_{0}=\left[25 c^{2}+(a-5 \phi)^{2}\right] a / 25 c(a-5 \phi)\right)$. The solid lines correspond to the Turing and Hopf bifurcations, crossing each other at the codimension-two point. A dashed line calculated from numerical simulations bounds from below the region of parameters where global (systemsize) oscillations are observed as final solution. Four different representative conditions are spotted in the figure. Leaving the stable region, we identify first, as shown in Fig. 1(b), a Turing instability giving rise to spots that pattern the medium steadily. Next we enter the region where mixed modes, in the form of oscillating spots, were reported [2] [see Fig. 1(c)]. Past but not far from the line denoted global-Hopf we 
(a)

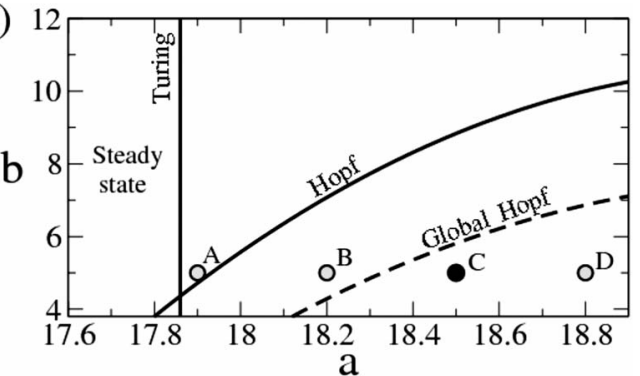

(b)

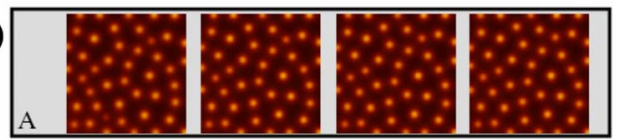

(c)

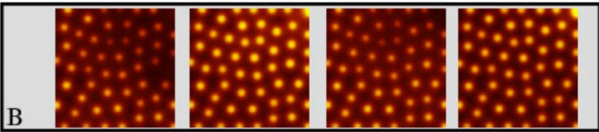

(d)

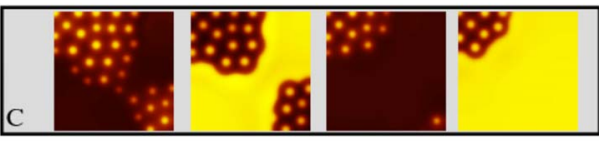

(e)

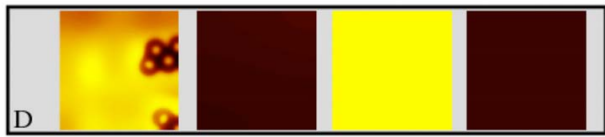

FIG. 1. (Color online) (a) Phase diagram of the LE model for fixed parameters $c=0.3, d=1.07$, and $\phi_{0}=3.2$. Vertical and curved solid lines correspond, respectively, to Turing and Hopf bifurcations. Dashed line distinguishes the region with global oscillations. Circles (black and gray) denote parameter values employed for the deterministic simulations with (b) $a=17.9$, (c) $a=18.2$, (d) $a=18.5$, and (e) $a=18.8$. For each evolution we show four snapshots corresponding to $t=50$ t.u., $t=100$ t.u., $t=150$ t.u., and $t=200$ t.u. Note that periods of oscillations are (c) $T_{0}=8.8$ t.u. (d) $T_{0}=7.3$ t.u., and (e) $T_{0}=8.5$ t.u. Bright and dark colors in the snapshots correspond, respectively, to high and low concentrations of the field $v$. Discretization units are $\Delta x=0.5$ s.u. and $\Delta t=0.0025$ t.u. in a grid of $64 \times 64$ s.u. ${ }^{2}$.

detect the conditions we will focus on hereafter: a Turinglike patterned structure, initially evolved from random initial conditions, looses stability and fades away as an advancing front of uniform oscillation progressively invades the system [see Fig. 1(d)]. Finally the most extreme region is reached where the oscillating regime undisputedly governs the whole system [see Fig. 1(e)].

The striking effect of spatially distributed fluctuations in stabilizing steady patterns over oscillatory modes is shown in Fig. 2. To better assess the phenomenon, the conditions are chosen such that we introduce, then transiently increase, and finally remove the noise. Simulations start with a random initial distribution of $u$ and $v$. The extended system transiently displays a Turing pattern, progressively replaced by a global oscillatory mode [Fig. 2(a)]. When external noise is introduced, the regularly oscillating region gradually collapses and spots reappear to eventually dominate the lattice entirely [Fig. 2(b)]. The spatiotemporal noise has thus converted a uniform oscillatory regime into a steady spatial pattern. If the strength of the noise is further increased the spatial order is destroyed and a totally uncorrelated behavior is

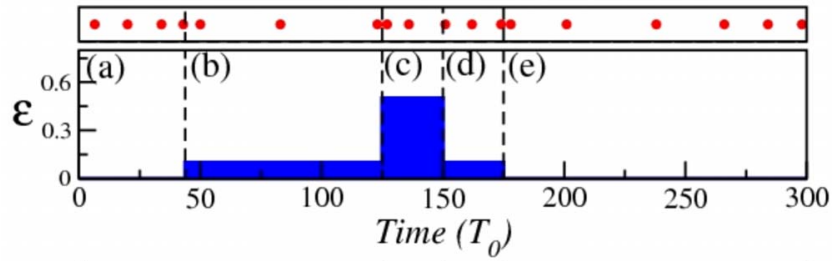

(a)

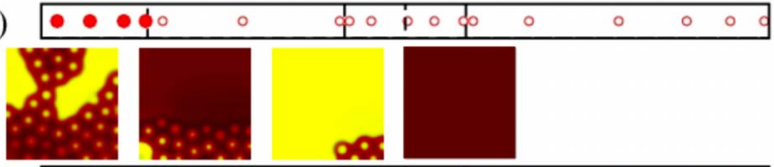

(b)

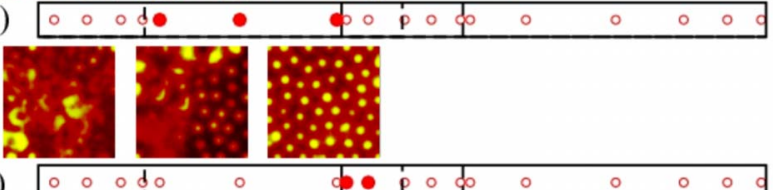

(c)

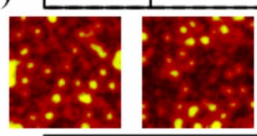

(d)
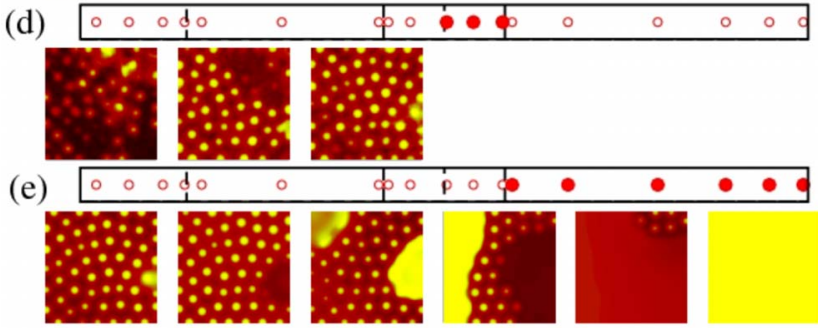

FIG. 2. (Color online) Stochastic simulation of the LE model in a situation of competition between Hopf (period $T_{0}=7.3$ t.u.) and Turing modes. Parameter values correspond to the black circle in Fig. 1 [see also Fig. 1(d)]. Top panel shows the evolution of the noise strength $(\epsilon)$ during the simulation. Top points indicate when the snapshots of the field $v$ are taken. Five different regions are studied in panels (a)-(e) corresponding to the regions noted in the top panel. (a) Four snapshots of the competition in absence of noise. (b) Three snapshots after switching on $\left(t=43 T_{0}\right)$ the noise with intensity $\epsilon=0.1$ and $\tau=0.1$. (c) Two snapshots after the increase in the noise to $\epsilon=0.5\left(t=125 T_{0}\right)$. (d) Three snapshots after the decrease to $\epsilon=0.1\left(t=150 T_{0}\right)$. (e) Six snapshots after switching off the noise $\left(t=175 T_{0}\right)$.

observed composed of heterogeneous oscillations and randomly appearing and disappearing spots in a noisy background [Fig. 2(c)]. The reduction in noise intensity permits the recovery of the steady spatial pattern [Fig. 2(d)]. The time needed for the development of the spatial pattern in this case is faster than in the case where the spatial pattern arose from global oscillations [Fig. 2(b)]. If the noise is finally switched off, global oscillations regain the whole system [Fig. 2(e)].

In the presence of low-strength fluctuations, the system dynamics is not affected and is dominated by global oscillations. Above a certain level of noise, conversely, there is no signature of spatial and temporal coherence as mentioned above. Between these two limits one expects to find an optimal noise strength which maximizes the spatial coherence of the pattern. To characterize this coherence enhancement, we calculate the spatial autocorrelation function $C(\lambda, t)$ $=\langle[v(x, t)-\bar{v}][v(x+\lambda, t)-\bar{v}]\rangle$. To eliminate any residual de- 

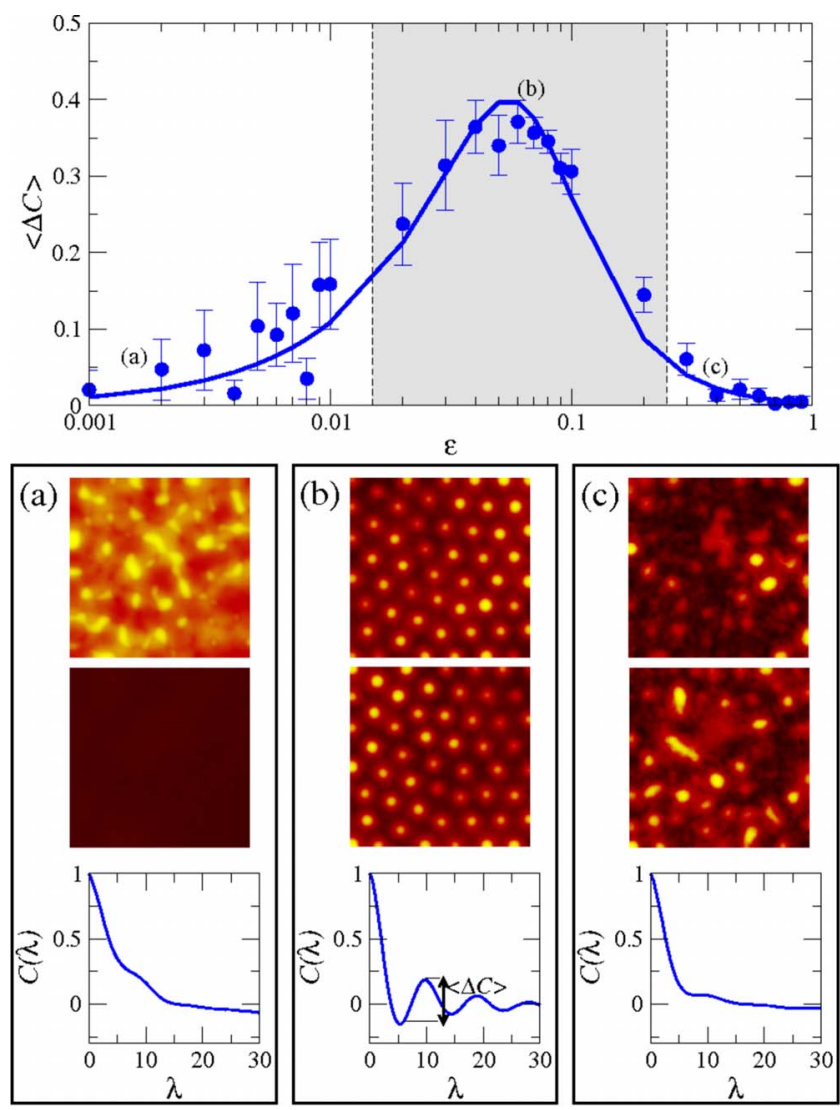

FIG. 3. (Color online) Dependence of the order parameter $\langle\Delta C\rangle$ on the intensity of the noise $(\epsilon)$ for $\tau=0.1$. Each point corresponds to an average over five realizations and the bars represent the error of the mean value. The line is a nonlinear fit (see text). Panels (a) - (c) correspond to (a) $\epsilon=0.002$, (b) $\epsilon=0.04$, and (c) $\epsilon=0.4$. Images on the panels are separated half period. The definition of the order parameter is sketched in panel (b).

pendence on the phase of the underlying oscillation we integrate such quantity over a period

$$
C(\lambda)=\int_{t}^{t+T} C(\lambda, t) d t .
$$

We further define a spatial-order parameter as the difference $\Delta C=C\left(\lambda_{0}\right)-C\left(\lambda_{m}\right)$ between the autocorrelation at its maximum whenever such characteristic length $\lambda_{0}$ exists and at its minimum for half such correlation length. Finally, we calculate the average $\langle\Delta C\rangle$ over several realizations.

In Fig. 3 we plot the dependence of the order parameter on the noise strength, keeping its correlation time constant. A maximum for an optimal noise strength $\epsilon_{o}$ markedly exists as a clear signature of a spatial stochastic coherence phenomenon, previously reported for pure Turing instability [11] and for spiral waves in excitable media [18]. Examples of particular calculations of $C(\lambda)$ are plotted in the three panels of Fig. 3.

In what follows we report a systematic study of the dependence of $\langle\Delta C\rangle$ on the noise strength $\epsilon$ and on the temporal correlation $\tau$. The first part of this Rapid Communication is summarized in Fig. 4(a), where dark (bright) regions rep-
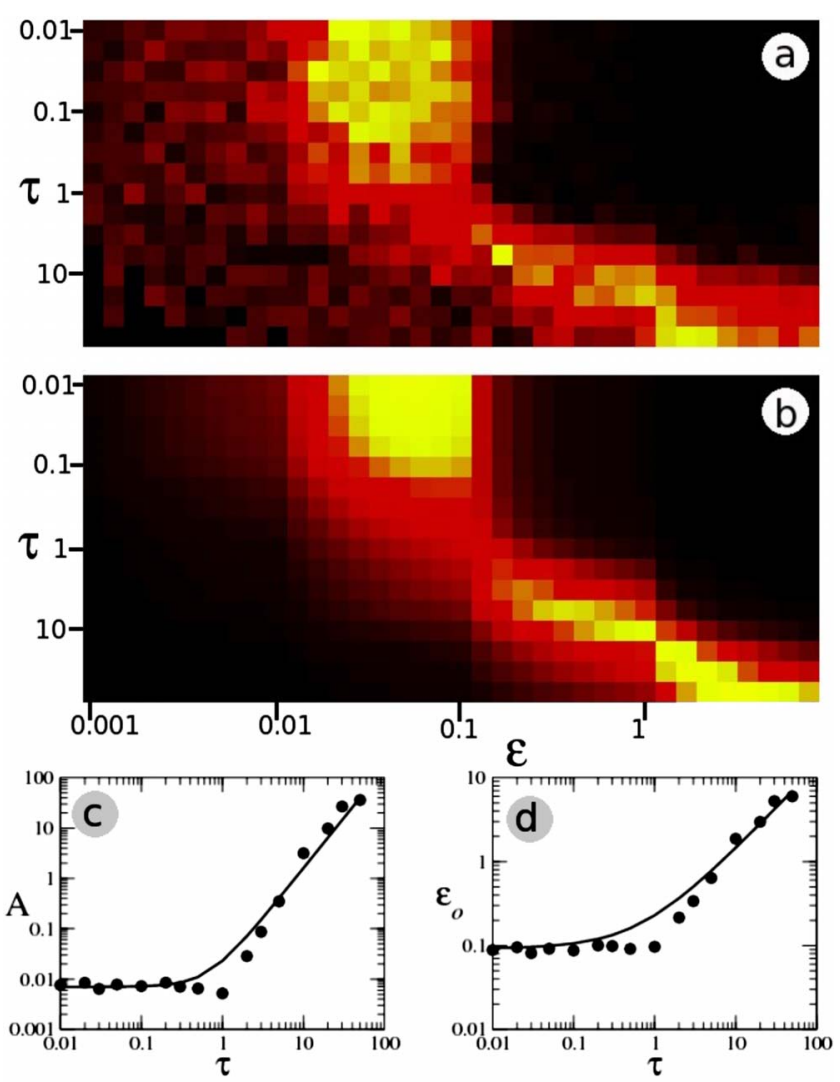

FIG. 4. (Color online) Dependence of the control parameter $\langle\Delta C\rangle$ on the intensity $(\epsilon)$ and the temporal correlation $(\tau)$ of the noise (a) for the numerical simulations and (b) for the phenomenological nonlinear function (4). Bright (dark) colors correspond to high (low) values of $\langle\Delta C\rangle$. [(c) and (d)] Results from the fitting using Eq. (4) of the numerical simulations. Continuous lines are the curves $A(\tau)$ and $\epsilon_{o}(\tau)$ (see text). Fitting parameters are $A_{o}=0.007$, $\epsilon_{o}^{o}=0.092, A_{\infty}=0.016$, and $\rho_{o}=0.137$. Note that scales of axes in panels (a) and (b) have been adapted and axis values of $\tau$ increase downwards.

resent small (large) values of the order parameter. There are clearly two different regimes: for low correlation times the dependence of the spatial coherence parameter on the noise strength is independent of $\tau$, while for large correlation times the optimal noise strength $\left(\epsilon_{o}\right)$ grows linearly with the correlation time, and an optimal noise intensity $\rho_{o}=\epsilon_{o} / \tau$ can be defined. In between, for $\tau \approx 1$, there is a crossover with a marked decrease in the value of $\langle\Delta C\rangle$. A power-law fit covers the whole range of studied conditions

$$
F(\epsilon, \tau)=\frac{2 A(\tau) \epsilon}{\epsilon_{0}(\tau)^{3}+2 \epsilon^{3}} .
$$

with $A(\tau)=A_{o}+A_{\infty} \tau^{2}$ and $\epsilon_{o}(\tau)=\epsilon_{o}^{o}+\rho_{o} \tau$ [see Figs. 4(c) and $4(d)]$. Interestingly with this unique function we can reproduce similarly well both the large and small correlation-time limits, as shown in Fig. 4(b), and even the minimum observed in the maximum coherence for intermediate values of $\tau$.

We have finally studied the effect of a correlation length of the noise by increasing the size of the pixel of noise. 
Results (not shown) indicate that as the spatial correlation is increased, the noise becomes less effective in obtaining a maximal coherence in the spatial pattern. In fact, for correlation lengths of the order of the size of the spots, the noise is not able to recover the static pattern anymore and global oscillations permanently dominate the system.

The numerical results reported here show a noise-induced enhancement of the stability of the spatial Turing mode against the temporal Hopf. At the parameter region here considered, the system when free of fluctuations transits from an initial (metastable) Turing pattern to a regime of homogeneous temporal oscillations. The introduction of spatiotemporal external fluctuations reverses such transition for moderate values of the noise strength and appropriate length and time correlation parameters.

Further away from the codimension-two point but under similar conditions, fluctuations do not rescue the Turing pattern but rather accelerate the appearance of the global oscillations. This observation reduces the generality of our results but not their relevance. It highlights the particular interaction among the noise and the two bifurcations close to the codimension-two point. Indeed, we can reasonably expect that both instabilities may display different susceptibilities to structured noise depending on the correlation parameters. Importantly enough the reversed stability is hardly observed when the correlation time is of the order of the natural time scale of the system (the period of the unforced oscillations), which points to a sort of resonant stabilization of the Hopf mode.
In general global oscillations must entrain synchronized areas of the spatially extended system and in that respect a low-amplitude Hopf mode should be rather unstable to additive fluctuations of small correlation length. Conversely, the Turing mode should not be able to filter distributed randomness of correlation larger than its intrinsic wavelength. As a matter of fact, we have checked that starting directly from Turing patterns close to the oscillatory regime no evidence of stability reversal by noise is detected except precisely when approaching the limit of large correlation lengths (homogenous fluctuations) where the Hopf mode again dominates the system. As a final remark let us stress that the present reported results have been observed strictly in 2D systems with no counterpart in one-dimensional (1D) realizations.

We hope that our analysis may stimulate new research on the selection of self-organization modes by noise. In particular a challenging question to investigate is whether the predominance of the Turing mode over the Hopf mode here reported is a generic feature of extended systems close to coexisting bifurcations or a rather peculiar outcome of the CDIMA system.

Financial support was provided by MEC (Spain) under Project No. FIS2006-03525 (F.S.), by DURSI though Project No. 2005-SRG/00653 (F.S.), and by the DFG (Germany) within the framework of SFB 555 "Complex nonlinear processes" (S.A). The authors acknowledge David Gómez Míguez, Sten Rüdiger, and Lutz Schimansky-Geier for stimulating discussions.
[1] M. C. Cross and P. C. Hohenberg, Rev. Mod. Phys. 65, 851 (1993).

[2] D. G. Míguez, S. Alonso, A. P. Muñuzuri, and F. Sagués, Phys. Rev. Lett. 97, 178301 (2006).

[3] A. Kaminaga, V. K. Vanag, and I. R. Epstein, J. Chem. Phys. 122, 174706 (2005).

[4] F. Sagués, J. M. Sancho, and J. García-Ojalvo, Rev. Mod. Phys. 79, 829 (2007).

[5] A. Mikhailov and K. Showalter, Phys. Rep. 425, 79 (2006).

[6] F. Sagués and I. R. Epstein, Dalton Trans. 7, 1201 (2003).

[7] S. Kádár, J. C. Wang, and K. Showalter, Nature (London) 391, 770 (1998).

[8] S. Alonso, I. Sendiña-Nadal, V. Pérez-Muñuzuri, J. M. Sancho, and F. Sagués, Phys. Rev. Lett. 87, 078302 (2001).

[9] P. S. Bodega, S. Alonso, and H. H. Rotermund, J. Chem. Phys. 130, 084704 (2009).
[10] A. Sanz-Anchelergues, A. M. Zhabotinsky, I. R. Epstein, and A. P. Muñuzuri, Phys. Rev. E 63, 056124 (2001).

[11] O. Carrillo, M. A. Santos, J. García-Ojalvo, and J. M. Sancho, Europhys. Lett. 65, 452 (2004).

[12] S. S. Riaz, S. Dutta, S. Kar, and D. S. Ray, Eur. Phys. J. B 47, 255 (2005).

[13] S. Alonso, D. G. Míguez, and F. Sagués, EPL 81, 30006 (2008).

[14] X. Sailer, D. Hennig, V. Beato, H. Engel, and L. SchimanskyGeier, Phys. Rev. E 73, 056209 (2006).

[15] A. P. Muñuzuri, M. Dolnik, A. M. Zhabotinsky, and I. R. Epstein, J. Am. Chem. Soc. 121, 8065 (1999).

[16] I. Lengyel and I. R. Epstein, Science 251, 650 (1991).

[17] J. García-Ojalvo and J. M. Sancho, Noise in Spatially Extended Systems (Springer, New York, 1999).

[18] M. Perc, Phys. Rev. E 72, 016207 (2005). 